\title{
Modelos de integración de la tecnología en la educación de personas que desempeñan funciones ejecutivas y de dirección: el TPACK y el SAMR
}

Technology Integration Models into adult education: TPACK and SAMR

\section{Volumen 21, Número 1}

Enero - Abril

pp. 1-27

Roy Campos Retana

Citar este documento según modelo APA

Campos Retana, Roy. (2021). Modelos de integración de la tecnología en la educación de personas que desempeñan funciones ejecutivas y de dirección: el TPACK y el SAMR. Revista Actualidades Investigativas en Educación, 21(1), 1-27. Doi. 10.15517/aie.v21i1.42411 


\title{
Modelos de integración de la tecnología en la educación de personas que desempeñan funciones ejecutivas y de dirección: el TPACK y el SAMR
}

Technology Integration Models into adult education: TPACK and SAMR

\section{Roy Campos Retana ${ }^{1}$}

\begin{abstract}
Resumen: La transformación digital supone uno de los principales retos educativos globales, los cuales se han visto acelerados por diversas tendencias del entorno actual y recientemente, por la pandemia del COVID-19. Aunque se han analizado los factores que restringen y habilitan la integración de la tecnología en entornos virtuales y presenciales, esas investigaciones se han enfocado principalmente en la educación de la niñez y de la juventud, pero no de personas adultas. Mediante un análisis de la teoría naciente (propone respuestas tentativas a nuevas preguntas) y madura (presenta construcciones y modelos bien desarrollados) de la educación de personas ejecutivas y los modelos de integración de la tecnología, este ensayo científico procura desarrollar una teoría intermedia que cierre la brecha entre ambos campos del conocimiento. Este estudio propone que los modelos de integración de la tecnología activan la reflexión del profesorado de personas que desempeñan funciones ejecutivas y de dirección. Se concluye que modelos, tales como el TPACK y el SAMR facilitan la reformulación de los modelos mentales para integrar la tecnología en la práctica docente, porque la mera adición de dispositivos o plataformas virtuales no garantiza un adecuado proceso de enseñanza-aprendizaje.
\end{abstract}

Palabras clave: formación docente, tecnología educacional, formación de personas administradoras.

Abstract: Global trends and COVID-19 have put the world into an educational challenge, boosting digital transformation. Although research studies had analyzed enablement's and constraints of technology integration through virtual and face-to-face contexts, their focus has been on K-12, high schools, and higher education, but not on adult education. Through an analysis of available theory, this scientific essay develops a moderate approach that fills the gap between technology integration and executive education. This theoretical paper suggests that cognizance of TPACK and SAMR -tech frameworks- encourage instructors to reflect and reframe their mental schemes and integrate technology into their teaching practice. This work also sustains that such integration requires a dedicated process beyond the mere addition of technologies in the educational environment.

Keywords: teacher education, educational technology, management education.

\footnotetext{
${ }_{1}$ Profesor de la Universidad de Costa Rica en la Escuela de Administración de Negocios, San José, Costa Rica. Master en Gobierno y Cultura de las Organizaciones por la Universidad de Navarra, Navarra, España, donde cursa sus estudios doctorales. Orcid https://orcid.org/0000$\underline{0001-7519-9219}$
}

Dirección electrónica: roy.camposretana@ucr.ac.cr

Ensayo recibido: 22 de junio, 2020

Enviado a corrección: 29 de setiembre, 2020

Aprobado: 9 de noviembre, 2020 


\section{Introducción}

En la última década, la integración de tecnologías en los procesos de enseñanzaaprendizaje se ha convertido en uno de los retos más relevantes de la educación en general (Hashim, Tan, y Rashid, 2015; Kopcha, Rieber, y Walker, 2016; Martin y Ertzberger, 2016). En un corto y mediano plazo, el escenario de esas tendencias tecnológicas supondrá retos sociales, tecnológicos, económicos y políticos para el personal docente, estudiantado y para las instituciones educativas como un todo (Brown et al., 2020, p. 7). Los principales obstáculos para acelerar la transformación digital de las instituciones educativas son: una insuficiente coordinación y planificación inter-institucional, poca inversión en tecnologías de avanzada y escasa comprensión sobre los beneficios potenciales del impacto transformador de esas tecnologías (Miller, 2019, p. 13). La pandemia del COVID-19 aceleró los procesos de transformación digital en el sector educativo y ha exigido a las organizaciones simplificar las prácticas institucionales, alinear y dar sostenibilidad a las inversiones tecnológicas y cambiar la forma en que se venía trabajando con el alumnado (Grajek, 2020, p. 8).

No obstante, existen numerosas barreras que limitan o inhiben la apropiada integración de la tecnología en los salones de clases tanto físicos como virtuales: las creencias del profesorado (Ertmer, Ottenbreit-Leftwich, Sadik, Sendurur, y Sendurur, 2012; Scott, 2013), la percepción y ansiedad sobre el uso de la tecnología (Kotrlik y Redmann, 2005; McClure, 2018), la complejidad de la tecnología a utilizar (Aldunate y Nussbaum, 2013; Tarling y Ng'ambi, 2016) y la percepción sobre la facilidad con que se usa (Howard, Chan, y Caputi, 2015; John, 2015). Uno de los principales problemas estriba en que se ha estudiado mucho cómo funciona la tecnología misma y no tanto cómo se integra a la educación (Caird y Lane, 2015; Mishra y Koehler, 2006, p. 1019). Desafortunadamente, esta problemática tan actual se ha analizado, sobre todo, en el campo de la educación de la niñez y la juventud (Harris, Mishra, y Koehler, 2009; Hilton, 2016), pero poco en el campo de la educación de personas que desempeñan funciones ejecutivas y de dirección² (Spector, Elen, Merrill, y Bishop, 2014).

Para contribuir a los retos globales de la educación, en las últimas décadas, se han desarrollado diversos modelos para integrar la tecnología en los procesos de enseñanza y aprendizaje. Los más reconocidos son: Arizona Technology Integration Matrix (TIM), Apple Classrooms of Tomorrow - Today (ACOT2), Technological Pedagogical Content Knowledge (TPACK) y Substitution Augmentation Modification Redefinition (SAMR). Debido a estudios

\footnotetext{
2 En adelante, se utilizarán indistintamente los términos personas ejecutivas, y personas que desempeñan funciones ejecutivas y de dirección.
} 
recientes que vinculan la reflexión del profesorado con una adecuada integración de la tecnología (Campos, 2020), este ensayo científico pretende profundizar en la teoría existente para entender la relación entre los modelos de integración de tecnología y la reflexión del profesorado (Anastopoulou, Sharples, y Baber, 2011; Gebre, Saroyan, y Bracewell, 2014; Verpoorten, Westera, y Specht, 2012). Bajo un enfoque conceptual de la educación de la tecnología en personas adultas, este ensayo científico pretende entender cómo la persona docente puede acelerar el proceso de integración de la tecnología con el uso de modelos como el TPACK y el SAMR, ya sea para hacerlo en modalidad presencial o virtual.

\section{Desarrollo del tema}

\subsection{Proposición: modelos de integración tecnológica en la reflexión docente}

Considerando que "la integración de la tecnología es la forma en que las capacidades y restricciones de la tecnología están incorporadas en el ejercicio profesional" (Campos, 2020, p. 25), este ensayo científico responde a la pregunta ¿Cómo los modelos de integración tecnológica (TPACK, SAMR) habilitan la reflexión del profesorado de personas ejecutivas? Para responderla se aplicará el concepto de la Gestalt a las clasificaciones de los objetivos del conocimiento, a Bloom y a la taxonomía revisada. Se entenderán los fundamentos del TPACK y luego se analizará el SAMR. Se relacionarán estos modelos entre sí y sus diversas aplicaciones a la educación de personas ejecutivas.

En cuanto al abordaje de este ensayo científico, Edmonson y McManus (2007, p. 1158) sostienen que la teoría de la investigación en la gestión y dirección de empresas recorre tres etapas: la teoría naciente, la intermedia y la teoría madura. Una teoría madura es aquella que presenta construcciones y modelos bien desarrollados, que han sido estudiados en el tiempo con precisión cada vez mayor, por una multiplicidad de académicos, dando por resultado un cuerpo consistente ampliamente reconocido y que constituye por tanto un saber acumulado. La teoría naciente, en cambio, propone respuestas tentativas a nuevas preguntas de cómo y por qué, sugiriendo a menudo nuevas conexiones entre fenómenos. La teoría intermedia, posicionada entre lo maduro y lo naciente, presenta explicaciones provisionales de los fenómenos, a menudo introduciendo una nueva construcción y proponiendo relaciones, entre ella y los constructos establecidos.

El presente ensayo científico procura desarrollar una teoría intermedia, tomando conceptos consolidados sobre el aprendizaje de los individuos, desarrollados a mediados del siglo $\mathrm{XX}$ y conectándolos con otras más recientes, de inicios del siglo $\mathrm{XXI}$, en torno a la 
integración de la tecnología. Esta perspectiva histórica será de algún modo evidente a lo largo de este documento, que procura poner en diálogo investigaciones maduras con las nacientes, tanto de la educación como de la tecnología. En resumen, se abordarán los fundamentos del aprendizaje audiovisual, la taxonomía del conocimiento y su relación con los modelos de integración tecnológica. Concretamente, este ensayo científico analizará el TPACK y el SAMR, porque son los modelos más difundidos en el campo educativo y, por tanto, los que cuentan con mayor potencial de aplicación en la enseñanza de personas ejecutivas.

\subsection{Argumentos para la discusión}

\subsubsection{El aprendizaje audiovisual}

A mediados del siglo XX, Edgar Dale publicó la primera edición del libro Audiovisual Methods in Teaching (1946). Dale era profesor en escuelas y colegios de Estados Unidos y presidente del Departamento de Instrucción Visual de la Asociación Nacional de Educación. Una de las principales inquietudes que le motivaron a profundizar en la enseñanza con recursos audiovisuales fue comprender cómo lograr que su alumnado recordara mejor y olvidara menos lo que les enseñaba en el aula. Sostenía que la educación es un proceso interactivo: de lo concreto a lo abstracto y viceversa (Dale, 1946). El mero uso de un recurso audiovisual no es educativo por sí mismo, sino que debe estar en función de los conceptos que se desean enseñar. Para que el profesorado haga un buen uso de la tecnología no es suficiente utilizarla, sino que se debe hacer una combinación de experiencias particulares, las cuales permitan aprender conceptos abstractos (Kolb y Kolb, 2011a; 2011b; Kolb, 2015).

Dale (1946, pp. 39-52) propone que una manera de lograr un aprendizaje permanente será mediante el uso de materiales audiovisuales que generen experiencias de aprendizaje, el cual se resumirá en una representación visual llamada el "Cono de la experiencia", también conocida como el "Cono del aprendizaje" (Ver Figura 1). Se agrega a la derecha de cada zona una breve explicación con ejemplos resumidos, expuestos por el mismo Dale a lo largo del libro.

Con el fin de ampliar lo anterior se explicará el proceso de aprendizaje inductivo, en el paso de lo concreto a lo abstracto, a partir de experiencias particulares: cómo lograr altos niveles de conocimiento a partir del uso de elementos concretos, de recursos y materiales en apariencia aislados. Se revisará la taxonomía de Bloom y a partir de ella se irán decantando algunos elementos de la Gestalt, hasta relacionar el uso de recursos audiovisuales con los modelos de integración de tecnología. 


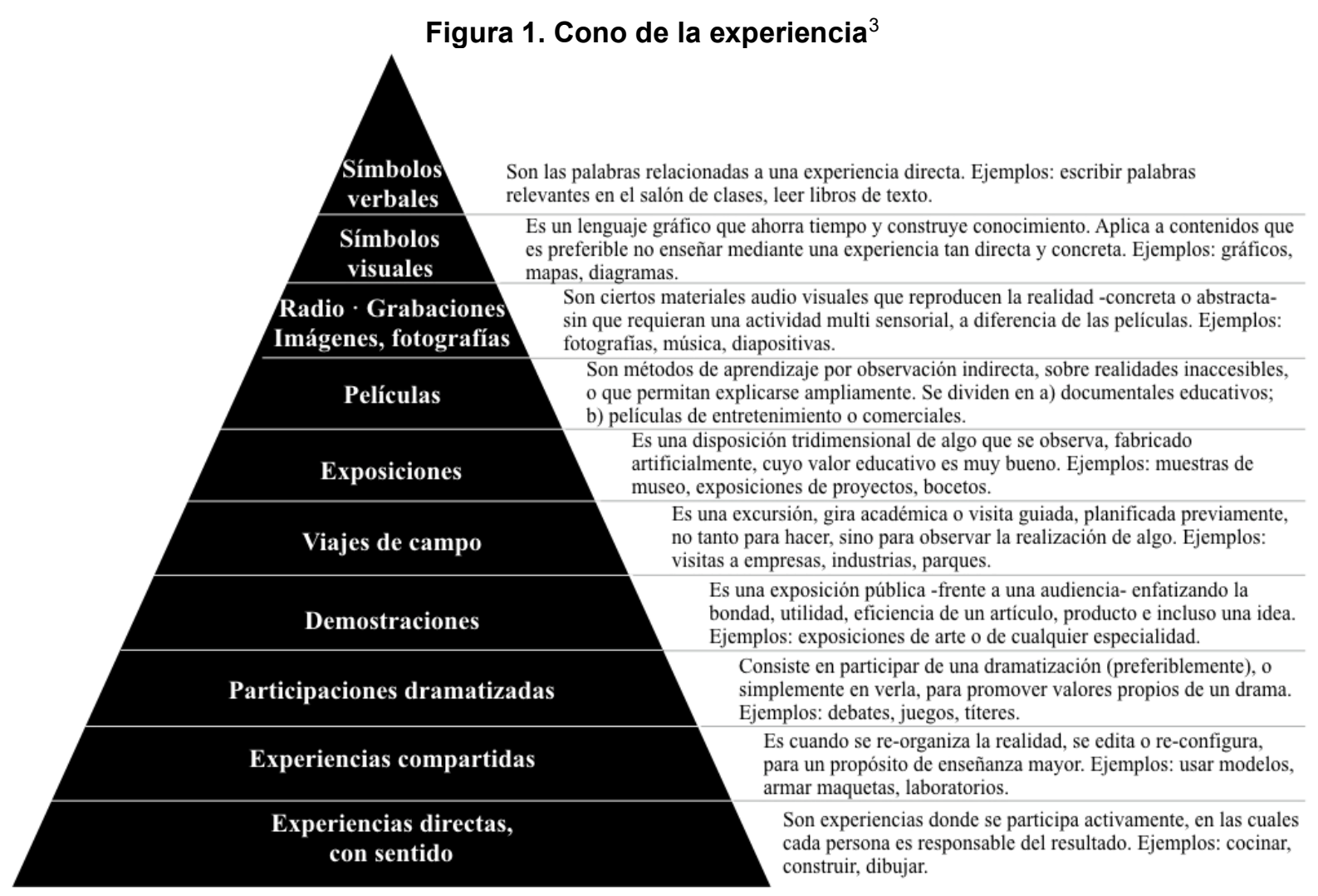

Fuente: Adaptado de Dale (1946, p. 68)

\subsubsection{Taxonomía del conocimiento}

En 1948 se celebró una reunión informal entre un grupo de evaluadores que asistían a la Convención de la Asociación Americana de Psicología (APA, por sus siglas en inglés). En esa sesión se expresó el interés de contar con un marco teórico que pudiera facilitar la comunicación entre los evaluadores a nivel nacional en Estados Unidos (Bloom, Engelhart, Furst, Hill y Krathwohl, 1956, pp. 4-16). Para efectos de este ensayo científico interesan dos de los integrantes de aquel comité, en particular, Bloom y Krathwohl.

Esta clasificación de objetivos cognitivos es probablemente la más conocida por sus categorías: conocimiento, comprehensión, aplicación, análisis, síntesis y evaluación. Estas seis categorías se subdividen a su vez en varias clases: esta subdivisión permite comprender

\footnotetext{
${ }^{3}$ Numerosos sitios de Internet ofrecen una imagen del mismo cono asignando porcentajes a cada zona, intentando defender su efectividad y proponiendo que el aprendizaje incrementa conforme se combinan las zonas o se desciende verticalmente en el cono. Fadel y Lemke (2012) desmienten que esas imágenes hayan sido elaboradas por Dale y las desmitifican, argumentando que no existe evidencia científica para comprobar tales índices de aprendizaje. Desafortunadamente, entre académicos y expertos en educación se sigue usando esa mítica figura, tan alejada de la realidad.
} 
cómo cada clase forma parte de un todo más complejo (Bloom et al., 1956). Ascender en esa subdivisión es lo que se entiende como conocimiento inductivo: se pasa de lo particular a lo general, de lo concreto a lo abstracto. Llevando esto a los modelos de integración hay que gestionar apropiadamente la incorporación de tecnologías en la enseñanza de conocimientos particulares para poder lograr el aprendizaje de conocimientos generales y abstractos.

La difusión de las taxonomías ha experimentado un proceso paulatino (Arneson y Offerdahl, 2018; Churches, 2009; Wang, Wei, Ding, y Li, 2017). De acuerdo con Krathwohl, en el momento en que se dio conocer la clasificación de Bloom, el ámbito educativo estaba poco familiarizado con el término "taxonomía" (Krathwohl, 2002, pp. 213-214). Cuarenta y cinco años después Krathwohl hizo una revisión de la taxonomía de Bloom y agregó un cambio importante a la última de las clases: sustituyó la evaluación por la creatividad (Anderson y Krathwohl, 2001; Dwyer, Hogan, y Stewart, 2014). La reclasificación Krathwohl es relevante en la innovación educativa (Gilboy, Heinerichs, y Pazzaglia, 2015). Esto puede apreciarse mejor en la Tabla 1, donde se compara la taxonomía revisada con la de Bloom. 
Tabla 1: Comparación entre la taxonomía de Bloom y la taxonomía revisada Taxonomía de Bloom Taxonomía revisada

1.0 Conocimiento.

1.10 Conocimiento de lo específico

1.11 Conocimiento de la terminología

1.12 Conocimiento de hechos específicos

1.20 Conocimiento de modos y sentidos de tratar lo específico.

1.21 Conocimiento de convencionalismos

1.22 Conocimiento de tendencias y secuencias

1.23 Conocimiento de clasificaciones y categorías

1.24 Conocimiento de criterio

1.25 Conocimiento de metodología

1.30 Conocimiento de universales y

abstracciones en un campo

1.31 Conocimiento de principios y generalizaciones

1.32 Conocimiento de teorías y estructuras

\subsection{Comprehensión}

2.10 Traducción

2.20 Interpretación

2.30 Extrapolación

\subsection{Aplicación}

\subsection{Análisis}

4.10 Análisis de elementos

4.20 Análisis de relaciones

4.30 Análisis de principios de organización

\subsection{Síntesis}

5.10 Producción de una comunicación única

5.20 Producción de un plan, o conjunto propuesto de operaciones

\subsection{Evaluación}

6.10 Evaluación en términos de evidencia interna

6.20 Juicios en términos de criterios externos
1.0 Recordar - recordar conocimiento relevante de la memoria de largo plazo

1.10 Reconocimiento

1.20 Recordación

2.0 Entender - Determinar el sentido en la instrucción de los mensajes, tanto oral, escrito como comunicación gráfica.

2.10 Interpretación

2.20 Ejemplificación

2.30 Clasificación

2.40 Resumen

2.50 Inferencia

2.60 Comparación

2.70 Explicación

3.0 Aplicar - Llevar el uso de un procedimiento a una situación dada.

3.10 Ejecución

3.20 Implementación

4.0 Analizar - Descomponer material en sus partes constitutivas y detectar cómo las partes se relacionan unas con otras, con una estructura o propósito.

4.10 Diferenciación

4.20 Organización

4.30 Atribución

5.0 Evaluar - Hacer juicios basados en criterios y estándares

5.10 Revisión

5.20 Crítica

6.0 Crear - Poner elementos conjuntamente para formar un todo novedoso y coherente, o hacer un producto original.

6.10 Generación

6.20 Planificación

6.30 Producción

Fuente: Adaptado de Bloom y Krathwohl (Bloom et al., 1956, pp. 200-207; Krathwohl, 2002, p. 214).

\subsubsection{Limitantes de la taxonomía e implicaciones para los modelos}

Una clasificación posee alcances valiosos, pero, como cualquier modelo, pretender acotar una realidad tan amplia, en este caso, una como lo es el proceso educativo, presenta limitaciones. Esa pretendida precisión querida por Bloom es la que despierta su principal problema: ¿cómo interpretar, en un grupo numeroso, cuál de las clases es la que está en 
ejercicio? (Bloom et al., 1956, pp. 15-16). Por ejemplo, si en una sesión con personas ejecutivas se pide elaborar un mapa conceptual, pero una de ellas lo hace aplicando los lineamientos que le da la persona docente mientras que otra lo hace de memoria, basándose en una experiencia similar que tuvo en el pasado, entonces estaríamos frente a dos clases distintas -conocimiento y aplicación- aun cuando la indicación que se les dio a ambos fue la misma.

Lo anterior desvela cuatro limitantes de esta clasificación. Primera, entender el valor de la experiencia. Solo es posible formar en conductas, especialmente las de personas adultas, si se toman en cuenta sus antecedentes, su bagaje. La formación de personas adultas es más efectiva cuando se aprovecha su experiencia personal y profesional. Segunda, el proceso de aprendizaje no es lineal. Las taxonomías asumen, en cierta medida, que el desarrollo de una categoría integra la anterior, pero esto en la realidad no siempre es así. Como se comentó en el ejemplo previo, una misma tarea puede requerir de distintas competencias: en un caso la aplicación de conceptos y en otra, el reconocimiento de antecedentes similares.

La tercera limitación es la clasificación de los resultados del aprendizaje y suponer que los comportamientos más complejos incluyen otros más simples. El resultado final dentro de un periodo de formación implica lograr resultados previos; por ejemplo, elaborar un mapa conceptual requiere un cierto nivel de creatividad. El resultado de aprendizaje es algo que se va decantando poco a poco, no un producto químicamente puro. Algo similar sucede con la integración tecnológica: los diferentes niveles pueden interactuar entre sí. La cuarta limitación es que las taxonomías, como modelos teóricos, entrañan un cierto nivel de complejidad, el cual es difícil de conciliar con la fluidez que requiere la docencia. Las divisiones entre las categorías de las taxonomías no son claras a la hora de ponerlas en práctica dentro del aula: son rígidas y, al mismo tiempo, difusas; de modo que para hacer viable la difusión de un modelo en el largo plazo deben hacerse esfuerzos para que su aplicación sea más fácil de conseguir en la actividad cotidiana de una persona docente.

Ante estas cuatro limitantes, Sousa (2015), especialista en neurociencia, opina que la revisión hecha por Krathwohl (2002) permite mejorar la calidad de la enseñanza y el aprendizaje del estudiantado. Entre las razones que ofrece están el hecho de que es un modelo más sencillo que otros, que requiere un modesto reentrenamiento de las personas docentes para entender la relación entre sus componentes. Si se extrapolan estas consideraciones a un entorno de integración tecnológica se puede afirmar que un aula, física 
o virtual, no requiere mayor inversión, sino simplemente aplicar los modelos de integración luego de un modesto reentrenamiento y acompañamiento.

\subsubsection{La Gestalt: taxonomías y modelos de integración tecnológica}

Para resolver estas limitantes de su clasificación, Bloom acude a la Gestalt (Bloom et al., 1956, p. 16). La Gestalt parte de que el todo es mayor que la suma de las partes: un comportamiento complejo es mayor que la suma de comportamientos más sencillos. Otra manera de entenderlo es considerar las conductas complejas como partes analizables en conductas más sencillas. Este movimiento de la psicología de la primera parte del siglo XX fue liderado por pensadores que influyeron considerablemente en diversos ámbitos del saber (Wagemans et al., 2012a; Wagemans et al., 2012b). Uno de sus principales autores fue Kurt Koffka.

Para el autor del libro Principles of Gestalt Psychology (Koffka, 1935), las partes se organizan dentro de un conjunto más complejo, el cual tiene sentido, y que permite que el todo esté organizado integralmente. Una taxonomía sigue un proceso inductivo: el conocimiento de la realidad va de lo particular a lo general. El planteamiento que se quiere hacer ahora es que los dos modelos de integración de tecnología a analizar (TPACK y SAMR) siguen esta lógica de clasificación: causa efecto, partes dentro de sub-todos que se llamarán clases o niveles, y al final habrá un todo funcional, que es la integración de conocimientos particulares en otros más abstractos. Entender realidades particulares, si están ordenadas lógicamente a un propósito de aprendizaje, permite encontrar un resultado de integración al final de ese camino.

La propuesta de este ensayo es que el uso de los modelos de integración de tecnología son un apoyo efectivo para lograr la integración dentro del aula de las personas ejecutivas, pues ayudan al profesorado a realizar un ejercicio reflexivo durante su acción docente. Para conseguirlo, antes se deben entender las leyes que gobiernan esas operaciones, las normas que rigen esos procesos, los principios controladores y la organización de esos principios (Polanyi, 1966, p. 34). Por eso, previo a la explicación de los modelos de integración en tecnología se revisarán dos conceptos de especial relevancia para el TPACK y que tendrán utilidad también para el SAMR: el conocimiento de los contenidos y el conocimiento pedagógico. Con ese fin se acudirá a Lee Shulman, miembro emérito de la Fundación Carnegie para el Fomento de la Enseñanza. 


\subsubsection{Conocimiento, contenidos y pedagogía}

En su artículo Those who understand: knowledge growth in teaching (Shulman, 1986), se distinguen tres categorías del conocimiento: el conocimiento de los contenidos, el conocimiento de la pedagogía de los contenidos y el conocimiento del plan de estudios. Se revisarán los dos primeros, que son los que atañen al análisis del TPACK.

El conocimiento de los contenidos se refiere a la cantidad de conocimiento y su organización en la mente de quien enseña (Shulman, 1986, p. 9). Taxonomías como la de Bloom son una de las muchas maneras de representar este tipo de conocimiento, pero pensar con precisión en el conocimiento de los contenidos requiere ir más allá de los hechos o conceptos propios de un área. Por ejemplo, el personal docente no solo debe ser capaz de enseñar las definiciones aceptadas en un campo, sino explicar por qué una proposición particular es aceptada y cómo se relaciona con otras proposiciones dentro y fuera de la disciplina, tanto en la teoría como en la práctica. Así es como la persona docente explica la importancia de la geo-localización de la flota vehicular de distribución debería ser capaz también de explicar sus implicaciones en términos financieros, de tiempo, competidores, riesgos, tecnología y sostenibilidad.

Por su parte, el conocimiento de los contenidos pedagógicos se orienta hacia la enseñanza de los contenidos (Shulman, 1986, p. 9), que incluye, para la mayoría de los temas que se enseñan en un área, las formas más usuales de representación de esas ideas, las analogías más poderosas, las ilustraciones, ejemplos, explicaciones y demostraciones habituales en el mundo, las maneras de representación y reformulación de contenidos que hacen esto comprensible a otros. El profesorado debe poseer un auténtico arsenal de formas alternativas de representación, algunas de las cuales habrán procedido de su investigación mientras que otras provendrán de las lecciones sugeridas por la práctica. Como se analizará a continuación, los modelos de integración ayudan a incorporar las capacidades y restricciones de los equipos tecnológicos en una clase física o virtual.

\subsubsection{Dos modelos de integración: TPACK y SAMR}

Los modelos de Punya Mishra y Matthew Koehler (2005), así como el de Ruben Puentedura (2006) se presentan el mismo año, pero con orientaciones distintas. Mishra y Koehler, académicos, construyen un modelo robusto desde el punto de vista teórico, pero quizá más difícil de implementar desde el punto de vista práctico, y que ha tenido mayor difusión en la educación superior. En cambio, Puentedura elabora un modelo sencillo, intuitivo, 
si se le puede calificar así, que carece de un aparato teórico sólido, pero con una interesante difusión en la educación primaria. Cada uno, con sus ventajas y desventajas, ha conseguido que personas docentes e investigadoras los aborden de manera creciente en sus aulas y estudios. Son escasas las referencias bibliográficas aplicando estos modelos a la enseñanza de personas ejecutivas, que es lo que se procurará ejemplificar durante la explicación de cada uno.

\subsubsection{TPACK}

EI TPACK -antes conocido como TPCK- se basa en las descripciones de Shulman sobre conocimiento de los contenidos pedagógicos para describir cómo estos interactúan con la comprensión de las tecnologías educativas y facilitar una enseñanza eficaz (Koehler y Mishra, 2009, pp. 61-62). Para estos autores, la palabra "tecnología" se aplica tanto a las analógicas como a las digitales, así sean nuevas o antiguas. Son conscientes de que muchas personas docentes recibieron su formación profesional en un momento en que la tecnología educativa se encontraba aún en una etapa de desarrollo incipiente. A esto se suma que el entorno educativo no siempre promueve la integración de la tecnología. Además, es frecuente que cuando el profesorado procura integrarla, la experiencia no siempre es la más positiva, sino que incluso resulta frustrante. Consideran entonces que no hay una mejor manera de integrar la tecnología en el plan de estudios. Por el contrario, los esfuerzos de integración deben ser creativamente diseñados y estructurados para entornos particulares y para los contextos específicos de cada aula (Baran, Canbazoglu Bilici, Albayrak Sari, y Tondeur, 2017; Chang, Jang, y Chen, 2015; Lee y Kim, 2014).

Los autores del TPACK consideran que los defensores de la integración en tecnología preveían cambios tan dramáticos que transformarían todos los procesos de enseñanza y aprendizaje; sin embargo, esto no ha sido así (Mishra y Koehler, 2006, p. 1018). El TPACK es precisamente un estudio de cómo integrar esa tecnología con el conocimiento de la pedagogía y de los contenidos desarrollados por Shulman. Este modelo se representa usualmente mediante un diagrama de Venn. Son tres círculos que se sobreponen, cada uno de los cuales representa un componente distinto del conocimiento del profesorado (Graham, 2011, p. 1954). El modelo incluye tres categorías básicas de conocimiento, como se puede observar en la Figura 2. 
Figura 2: Modelo TPACK y sus componentes

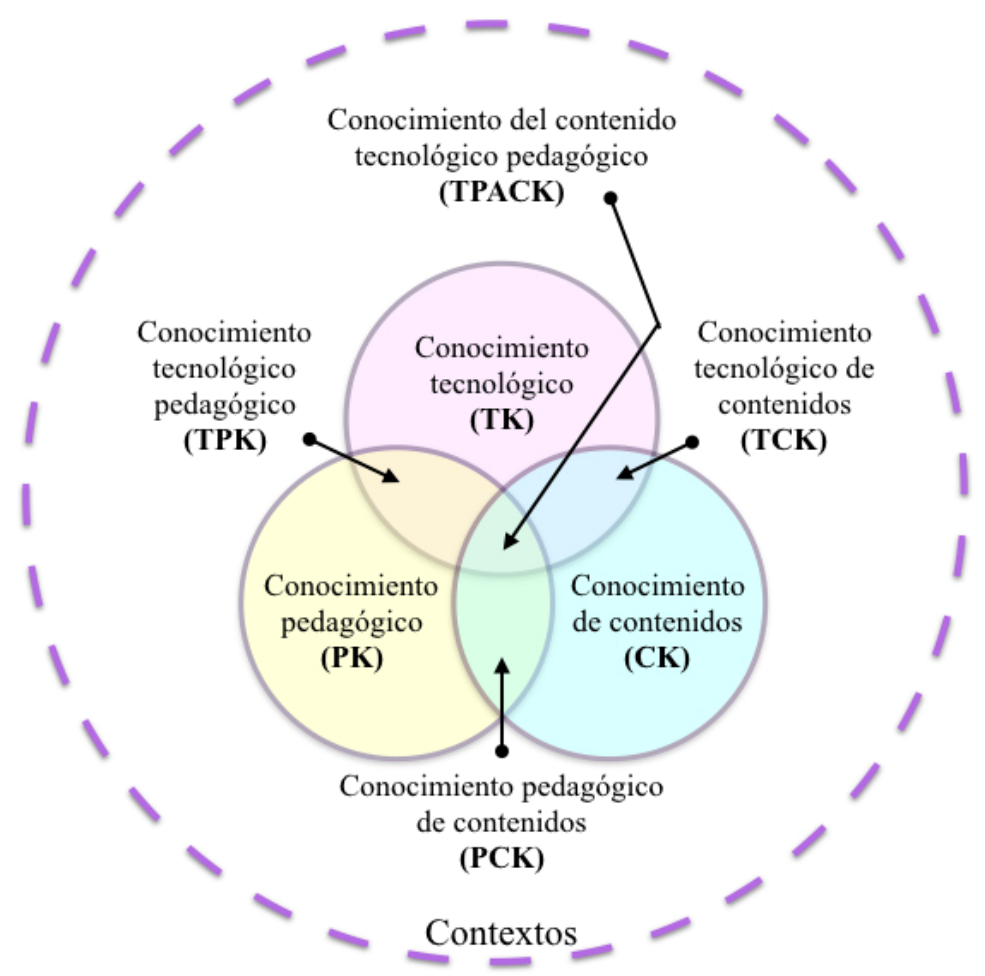

Fuente: Adaptado de Mishra y Koehler (2009, p. 63)

Para la elaboración de este diagrama en español se han conservado las siglas en inglés, así se evita generar confusiones con el diagrama y modelo originales ${ }^{4}$. A continuación, se explica cada sección del modelo, según Mishra y Koehler (2006):

El conocimiento de contenidos (CK) es la comprensión que el profesorado posee del tema que debe ser aprendido o enseñado (Koehler y Mishra, 2009, p. 63; Mishra y Koehler, 2006, p. 1026). Como afirmaba Shulman (1986), incluye conocer hechos, conceptos, teorías, procedimientos, esquemas organizativos, conexiones de ideas, conocimiento de evaluaciones y enfoques preestablecidos para desarrollar ese conocimiento. No contar con una base para aclarar y ampliar este tipo de conocimientos puede condicionar el trabajo futuro, también del estudiantado, debido a eventuales esquemas preconcebidos y con frecuencia errados; es decir, lo que con facilidad inducirá a nuevos errores a la hora de desarrollar otros conceptos en el área propia de contenidos de conocimientos pedagógicos (PCK).

\footnotetext{
${ }^{4}$ Cejas León, Navío Gámez y Barroso Osuna (2016, p. 109) prefieren utilizar el término en español competencias en lugar de conocimientos, puesto que "disponer de un conjunto de conocimientos, procedimientos y actitudes no supone ser competente". Aunque en este ensayo científico no se abordará esa discusión, la traducción de los autores se utilizó como referencia para precisar la traducción al castellano de los componentes del TPACK. Por ejemplo, en la adaptación de la figura 2.
} 
Por ejemplo, para una persona docente en negociación, conforman el CK los conceptos de negociación competitiva y colaborativa, los conflictos y su manejo, estilos, tácticas y estrategias de negociación. Son todos aquellos conceptos que se refieren a los fundamentos de la negociación interpersonal. Nótese que, en este momento, parece no haber relación clara con la tecnología. Precisamente por eso, la referencia a Shulman era esencial en el análisis de este modelo: si no se hacen explícitos los conceptos que encierran los contenidos nunca se podrá saber cómo integrarlos con la tecnología. Así es como se entiende lo dicho antes sobre las taxonomías: abordar las realidades sencillas para luego irlas integrando hasta crear conceptos más generales y abstractos (Shulman, 1986).

El conocimiento pedagógico (PK) apunta a una profunda comprensión por parte del profesorado de los métodos y de los procesos de enseñanza y aprendizaje (Koehler y Mishra, 2009, p. 64; Mishra y Koehler, 2006, p. 1026). Abarca, entre otros aspectos, propósitos, valores y objetivos educativos en general. El profesorado que posee un profundo conocimiento pedagógico entiende cómo el estudiantado conforma conocimientos, adquiere destrezas y desarrolla hábitos mentales y disposiciones positivas para el aprendizaje.

Así, entendido el PK, las posibilidades pedagógicas para que el profesorado desarrolle competencias de negociación en ejecutivos pueden estar facilitadas por métodos de enseñanza, tales como juegos de roles, casos o test. Pueden discurrir desde las dinámicas más sencillas que realice quien facilite la sesión, interactuando con el estudiantado, hasta aquellas otras más complejas que impliquen preparar o simplemente aplicar simulaciones, es decir, dinámicas donde las contrapartes poseen alguna información compartida y otra parte es confidencial. Es importante, a este respecto, notar de nuevo que en este punto la tecnología pareciera tampoco estar presente, pero se trata de hacer explícita la complejidad de la pedagogía, la cual fácilmente se descuida cuando se imparten clases con apoyos tecnológicos. Estas distinciones ayudan a la persona docente a evitar el uso de la tecnología de manera irreflexiva.

El conocimiento tecnológico (TK) se refiere a la comprensión por parte del profesorado de las tecnologías estándares, como libros, tiza y pizarra, y otras más avanzadas, como Internet y video digital (Koehler y Mishra, 2009, p. 64; Mishra y Koehler, 2006, p. 1027). Este conocimiento requiere las destrezas necesarias para operar tecnologías particulares. La adquisición de TK posibilita un sinnúmero de tareas al utilizar las tecnologías de la información. También habilita modos diferentes de llevar a cabo una tarea determinada. Esta 
conceptualización del TK no plantea un "estado final de integración", sino que lo concibe como algo evolutivo, abierto a la interacción y a la generación de tecnología.

Por ejemplo, una persona docente en negociación que integra la tecnología, usará videos ilustrativos sobre negociación y proyectará esquemas. O bien, si se trata de una ejemplificación más compleja, usará una plataforma virtual para recopilar los resultados de los ejercicios: los digitará en una hoja de cálculo para luego compartirlos a los demás participantes.

El conocimiento pedagógico de contenidos (PCK) consiste en saber qué métodos de enseñanza se ajustan a los contenidos, e igualmente entender cómo se pueden ajustar esos mismos contenidos en orden a una mejor enseñanza; es decir, poder realizar la integración de la pedagogía y los contenidos (Koehler y Mishra, 2009, p. 64; Mishra y Koehler, 2006, p. 1028). Consiste, por tanto, en la transformación del tema a enseñar, que ocurre en la medida en que el profesorado interpreta el contenido y encuentra múltiples maneras de representarlo, al mismo tiempo que adapta y ajusta los materiales educativos en formas variadas antes de enseñárselos a sus estudiantes.

Se actualiza este conocimiento cuando, en el curso de una simulación de negociación, quien facilita la sesión les orienta para utilizar tácticas de cierre, de bloqueo, de persuasión. De esta manera integra el temario con la metodología, al sugerirles también algunas técnicas, como el fingimiento, el atosigamiento, el desinterés, la empatía. Se les puede animar a hacer de policía bueno y policía malo, sin descuidar la relación y el resultado de la negociación. De esta forma es como los contenidos y la pedagogía se sobreponen.

El conocimiento tecnológico pedagógico (TPK) se plantea como la comprensión, por parte del profesorado, de cómo la enseñanza y el aprendizaje se optimizan al utilizar tecnologías particulares en modos específicos (Koehler y Mishra, 2009, p. 65; Mishra y Koehler, 2006, p. 1028). Esto incluye conocer las posibilidades pedagógicas y las limitaciones de una determinada gama de herramientas tecnológicas para después relacionarlas entre sí. En otras palabras, sucede cuando tecnología y pedagogía se integran eficazmente. Este conocimiento requiere una comprensión siempre actualizada de las limitaciones y posibilidades de las tecnologías a medida que se usan en los contextos, ámbitos de enseñanza y aprendizaje, y viceversa.

La persona docente en negociación tendrá, entonces, que poseer un cierto conocimiento de aquellos instrumentos que funcionen más ágilmente en términos de manipulación, transferencia de archivos, proyección y resolución de imágenes. Podría ser una cámara, un 
teléfono celular o una tableta para hacer filmaciones de los ejecutivos durante el proceso de negociación y luego transferirlo a un disco duro. Los autores sugieren especificar el tipo de tecnología que se usará. Y esto no es excepcional en la educación de personas adultas, aunque seguramente será mucho menor el nivel de complejidad que se dé entre un curso de ejecutivos y otro de tecnologías de información, por ejemplo.

El conocimiento tecnológico del contenido (TCK) radica en la comprensión, por parte del profesorado, de la reciprocidad en que tecnología y contenido están relacionados (Koehler y Mishra, 2009, p. 65; Mishra y Koehler, 2006, p. 1028). Es entender cómo se habilitan y se limitan. En otras palabras, consiste en la integración de tecnología y contenidos. Las personas docentes deben ir más allá del tema que enseñan: deben tener una comprensión profunda de la manera en que los contenidos o las diversas representaciones puedan ser transformadas por la integración de tecnologías particulares.

Cuando quien facilita la sesión ha descargado las filmaciones y fotografías de las personas ejecutivas mientras estas negociaban, podría analizar la comunicación y el lenguaje no verbal que han empleado entre ellas, el ambiente físico y los obstáculos de comunicación. Con el fin de identificar cuándo un gesto del rostro expresa desinterés o un posible engaño o bien un "falso-positivo", tendrá que haber realizado tomas cercanas, no solamente de la postura corporal en general, para identificar si esa posición manifestaba empatía o tensión, sino también para interpretar los gestos del rostro, por ejemplo.

El conocimiento del contenido tecnológico pedagógico (TPACK) es la integración entre contenido, tecnología y pedagogía: es una forma emergente de conocimiento que va más allá de los tres componentes "básicos" (Koehler y Mishra, 2009, p. 65; Mishra y Koehler, 2006, p. 1028). Al conseguirlo de manera simultánea, las personas expertas lo pondrán en ejecución de manera efectiva cada vez que deban impartir lecciones. De hecho, uno de sus principales desafíos será enfrentar los problemas educativos básicos y rediseñar la enseñanza de manera que logren un balance adecuado entre los tres elementos fundamentales. Se ha llegado aquí al ámbito de la complejidad y de la abstracción.

Para seguir con el escenario que se viene empleando, ahora, quien facilita proyecta las imágenes y videos que ha capturado, en las simulaciones de negociación, para proporcionar un feedback a las personas ejecutivas sobre el desarrollo de su competencia negociadora: compartirá fotografías para analizar el contacto visual, las posiciones de las manos y posturas. Analizará videos cortos para analizar la postura de los pies, la sonrisa o seriedad del semblante, las actitudes de desinterés, las miradas reflexivas o creativas y hará pausas para 
captar cuándo hay duda, tensión, escucha activa o pasiva, actitudes de colaboración o de competencia, de humor, tensión o rechazo. Es en este punto cuando contenidos, pedagogía y tecnología se sobreponen, es el momento de la integración.

Pese a las críticas al TPACK (Graham, 2011, p. 1953), las cuales pueden obedecer a que se trata de un modelo relativamente reciente y en proceso de difusión, se posee un aparato conceptual robusto en comparación con los demás. De hecho, el otro modelo que se analizará, el SAMR, apenas cuenta con alguna literatura informal que lo sustente y, para reafirmar lo anterior, casi ninguna literatura académica que lo aborde.

\subsubsection{2. $S A M R$}

Según Hamilton, Rosenberg y Akcaoglu (2016) el modelo SAMR, representado como una escalera, es un enfoque de cuatro niveles para seleccionar, usar y evaluar la tecnología en la educación primaria. Esa pirámide ascendente anima al profesorado a incorporarse desde los niveles inferiores hasta los más altos, lo que, según Puentedura (11 de diciembre, 2014), conduce a niveles más elevados de enseñanza y aprendizaje. Esta característica del SAMR lo hace más afín a la clasificación de una taxonomía que, como se revisó en páginas anteriores, pasa de lo concreto a lo abstracto, ascendiendo en nivel de complejidad, en sentido vertical. De hecho, Puentedura (11 de diciembre, 2014) ve el SAMR como una herramienta a través de la cual se pueden describir y categorizar los usos de la tecnología por parte de los maestros de educación primaria.

A diferencia del TPACK, que es complejo y en ocasiones difícil de distinguir entre sus áreas, el SAMR goza de tanta simplicidad que su comprensión resulta más sencilla. Mientras que el TPACK nace como un modelo de educación sobre la tecnología misma, lo que explica el énfasis que de esta se hace en ella, el SAMR es un modelo que fue diseñado para escuelas, con lo cual facilita elaborar procesos didácticos creativos (Aiyegbayo, 2015; Glover, Hepplestone, Parkin, Rodger, e Irwin, 2016; Lindsay, 2016).

Tal y como se explicó en el desarrollo de una taxonomía, una de las características fundamentales del SAMR es que la parte inferior corresponde a los niveles más elementales de la enseñanza de la tecnología, y conforme asciende en la escala llegará hasta los más complejos. Si en el TPACK esa complejidad se veía en términos concéntricos, en el SAMR la observaremos en sentido vertical, al igual que en Bloom. Esa complejidad se expresa en cuatro niveles (Puentedura, 11 de diciembre, 2014), los cuales se agrupan a su vez en dos categorías. Los niveles de sustitución y aumento corresponden a la categoría de mejora, mientras que los 
niveles de modificación y redefinición corresponden a la categoría de transformación (Ver Figura 3). En las categorías más básicas, el profesorado sigue siendo el centro de la enseñanza, mientras que en las últimas dos se convierte en una persona que facilita el proceso de aprendizaje.

Figura 3: Modelo SAMR y sus componentes

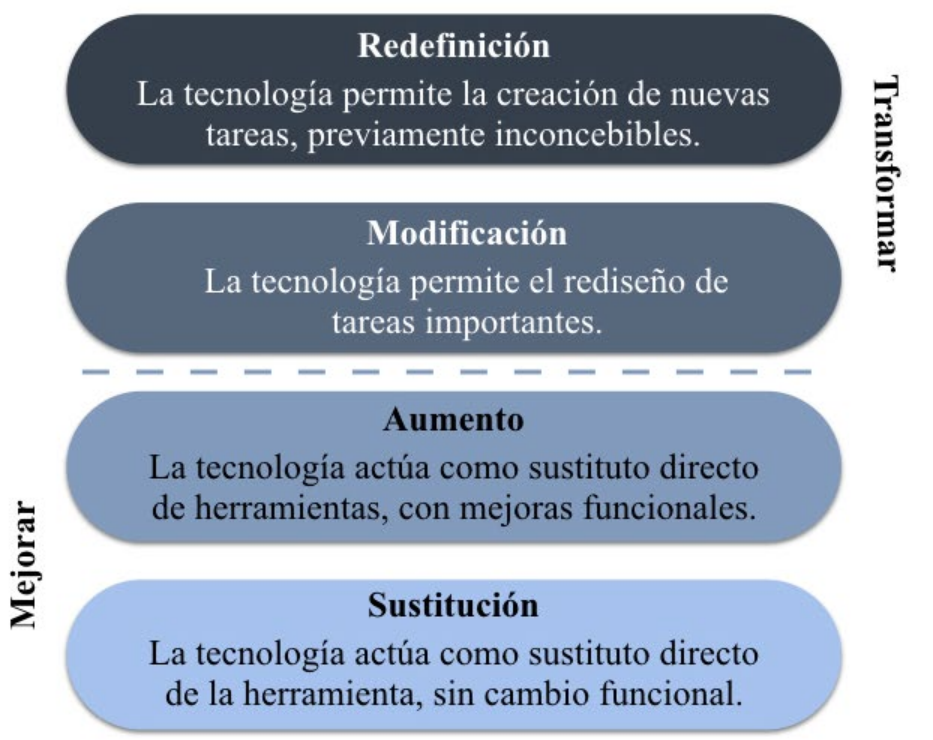

Fuente: Adaptado de Puentedura (8 de diciembre, 2010, p. 3)

A continuación, se explicarán cada uno de los niveles:

En el nivel de sustitución (Puentedura, 2006), la tecnología analógica se reemplaza por la tecnología digital, pero la sustitución no genera ningún cambio funcional. Según López García (2015), el profesorado integra la tecnología en sus actividades de enseñanza y aprendizaje de manera que el alumnado efectúa las mismas tareas que antes realizaba sin el soporte de la tecnología. La didáctica de las actividades se conserva, pero incorporar la tecnología puede favorecer la disposición del estudiantado para el aprendizaje de un tema. En este primer nivel, la persona docente es quien guía todos los aspectos de la clase apoyándose en la tecnología.

Para contrastar ambos modelos, se usará de referencia el ejemplo anterior: el taller de negociación. En un momento determinado se dijo que quien facilita recibiría en un papel los resultados de las negociaciones entre las partes, y que serían compartidos posteriormente para que todos los miembros del grupo pudieran verlos. La sustitución se da cuando se transcribe, en la hoja electrónica de cálculo, los resultados de esas negociaciones para compartirlos en una plataforma virtual. Se cumplen, entonces, todos los rasgos citados 
anteriormente: la didáctica se conserva, la disposición de los aprendices se favorece y la persona docente es quien les guía. No hay cambio funcional: solo se reemplaza la tecnología analógica por la digital.

En el nivel de aumento (Puentedura, 2006), la tecnología se intercambia y la función de la tarea o herramienta cambia positivamente de alguna manera. Es decir, la tecnología actúa como herramienta sustituta directa y sí genera una mejora funcional. López García (2015) comenta que si no se utiliza la tecnología la actividad de clase no sufrirá cambios drásticos en su diseño. En este segundo nivel del modelo tampoco se presentan cambios en la didáctica de las actividades. El uso de la tecnología pasa a manos del estudiantado de una manera mucho más activa que en el nivel anterior, porque ya no es el profesorado quien realiza las tareas propuestas, sino que ahora es responsabilidad del estudiantado efectuarlas.

Continuando con el caso previo, se trataría de una actividad en la cual las personas ejecutivas tengan una mayor participación y la tecnología genere un cambio positivo. Pueden ser las negociaciones con iteraciones, aquellas donde se requiere negociar una y otra vez. Por ejemplo, el "dilema del prisionero", que puede servir para demostrar el valor de la confianza en las negociaciones y explicar la frontera eficiente de Pareto, luego de hacer varias rondas en torno a una misma decisión: delatar al amigo o no delatarlo. Si ahora las contrapartes transcriben su decisión en la hoja de cálculo en la nube y esto les permite acumular los resultados de las decisiones previas se estaría generando un cambio funcional. Eso mismo se podría haber hecho presencialmente, cara a cara, y por eso se dice que la didáctica no cambia. Sin embargo, en la medida en que realizan tareas concretas, las personas participantes se involucran más y el resultado es más favorable que si no hubiese tecnología, entonces hay un aumento. Incluso, virtualmente, todos pueden conocer los resultados de sus decisiones en tiempo real, algo que antes no sucedía.

En el nivel de modificación (Puentedura, 2006), se rediseña el proceso de enseñanza para que la tecnología genere un valor añadido al aprendizaje; es decir, la integración tecnológica requiere un rediseño significativo de las tareas y la sesión para hacer un empleo efectivo de ella. Según López García (2015), el profesorado debe reformular las actividades de aprendizaje de modo que puedan integrar la tecnología, adaptándola a los recursos disponibles, según el enfoque e intención educativa que desee.

Tal como se explicó anteriormente, el lenguaje no verbal se puede mostrar con análisis de videos grabados y fotografías de quienes participan en las simulaciones o juegos de roles. Esto genera un valor agregado a la enseñanza, porque no basta con transcribir los resultados 
de una negociación, sino que hay un énfasis en analizar el proceso mismo de negociar. Es una competencia que, además, no se puede medir en todas sus dimensiones, porque implica variables intangibles, tales como el estilo personal de relacionarse y el manejo de posturas corporales para empatizar o intimidar. Que las personas participantes negocien mediante video llamadas y las graben para luego compartirlas a quien facilita la sesión, de modo que mejore el análisis para hacer en grupo, es un ejemplo de modificación.

Por último, el nivel de redefinición (Puentedura, 2006) se logra cuando la tecnología se utiliza para crear nuevas tareas, antes imposibles de lograr sin su mediación. Puentedura les llama "nuevas tareas, previamente inconcebibles (Diapositiva 3)”. López García (2015) sugiere que el profesorado se pregunte cómo puede la tecnología hacer posible una nueva actividad, antes inconcebible, y proponerse, así también, elaborar nuevos productos informáticos, en donde la colaboración entre el estudiantado y la tecnología será fundamental, por tanto, el vehículo de comunicación.

¿Qué pasa entonces si se pide al estudiantado que grabe y analice negociaciones de la vida real? Se mencionó que la intención de grabar a una persona ejecutiva mientras negociaba tenía como fin exponer al grupo su desempeño no verbal, incluso sin sonido. Lo que se plantea ahora es que sea el estudiantado quien analice ese lenguaje no verbal de sus compañeros. Pueden hacerlo mientras efectúan las simulaciones dentro del aula, incluso también fuera de ella, en modalidad virtual. Podrían grabar algunas de las negociaciones durante el ejercicio de su trabajo y editar videos de situaciones reales -durante una Junta Directiva, una sesión de ventas o una reunión con clientes internos de la organización- para pedirles que analicen los aspectos estudiados hasta el momento: técnicas de cierre y persuasión, actitudes colaborativas o competitivas, gestión de la confianza (la intensidad de la mirada, su tiempo y fijación, por ejemplo) y tantas otras posibilidades que van desde el manejo del humor hasta el control de la ansiedad (movimiento de las manos y posturas informales).

En las primeras dos etapas del SAMR se genera una mejora en el proceso didáctico gracias a la incorporación de la tecnología, pero en las últimas dos fases se crea una transformación de la enseñanza tradicional, y el profesorado deja de ser protagonista. Por otra parte, es interesante poder complementar los dos modelos: en el TPACK se desagregaban los principales componentes para luego integrarlos en una actividad concreta; en el SAMR se pueden identificar, a modo de escalones, los niveles de complejidad. Puentedura (2006) ha procurado relacionar el SAMR con el TPACK y la taxonomía de Bloom revisada. Esas relaciones se han intentado plasmar en la Tabla 2. 
Tabla 2: Relaciones del SAMR con TPACK y taxonomía revisada

\begin{tabular}{|c|c|c|c|c|}
\hline $\begin{array}{l}\text { Nivel del } \\
\text { SAMR }\end{array}$ & $\begin{array}{c}\text { Niveles } \\
\text { Tecnológicos } \\
\text { del SAMR }\end{array}$ & $\begin{array}{c}\text { Ejemplos } \\
\text { del SAMR en clase }\end{array}$ & $\begin{array}{c}\text { Relación } \\
\text { del SAMR } \\
\text { con el } \\
\text { TPACK }\end{array}$ & $\begin{array}{l}\text { Relación } \\
\text { SAMR con } \\
\text { taxonomía } \\
\text { revisada }\end{array}$ \\
\hline Redefinición & $\begin{array}{l}\text { La tecnología permite la } \\
\text { creación de nuevas tareas, } \\
\text { previamente inconcebibles. }\end{array}$ & $\begin{array}{lr}\text { Herramientas para la } \\
\text { visualización } & \text { de } \\
\text { aspectos narrativos y } \\
\text { estructurales del texto }\end{array}$ & TCPK & $\begin{array}{l}\text { Crear. } \\
\text { Evaluar. }\end{array}$ \\
\hline Modificación & $\begin{array}{l}\text { La tecnología permite el } \\
\text { rediseño de tareas } \\
\text { importantes. }\end{array}$ & $\begin{array}{l}\text { Herramientas textuales, } \\
\text { visuales y auditivas para } \\
\text { la construcción de } \\
\text { conocimientos } \\
\text { compartidos. }\end{array}$ & $\begin{array}{l}\text { TPK, } \\
\text { TCK, } \\
\text { PCK }\end{array}$ & $\begin{array}{l}\text { Evaluar. } \\
\text { Analizar. }\end{array}$ \\
\hline Aumento & $\begin{array}{l}\text { La tecnología actúa como } \\
\text { sustituto directo de } \\
\text { herramientas, con mejoras } \\
\text { funcionales. } \\
\text { Se utilizan funciones } \\
\text { básicas }\end{array}$ & $\begin{array}{l}\text { Diccionarios, guías de } \\
\text { estudio, sitios de historia } \\
\text { vinculados al texto en } \\
\text { línea. }\end{array}$ & $\begin{array}{l}\text { PK, } \\
\text { CK, } \\
\text { TK }\end{array}$ & $\begin{array}{l}\text { Aplicar. } \\
\text { Entender. }\end{array}$ \\
\hline Sustitución & $\begin{array}{l}\text { La tecnología actúa como } \\
\text { sustituto directo de la } \\
\text { herramienta, sin cambio } \\
\text { funcional. }\end{array}$ & $\begin{array}{l}\text { Textos de Shakespeare } \\
\text { leídos en versiones en } \\
\text { línea }\end{array}$ & $\begin{array}{l}\mathrm{P}, \\
\mathrm{C}, \\
\mathrm{T}\end{array}$ & $\begin{array}{l}\text { Entender. } \\
\text { Recordar. }\end{array}$ \\
\hline
\end{tabular}

Fuente: Adaptado de Puentedura (2006, pp. 3-4; 2014, pp. 7-20)

\section{Reflexiones finales}

La adopción de la tecnología en personas docentes se ve influenciada por numerosos aspectos, principalmente por sus creencias personales sobre la innovación en la enseñanza, así como por sus esquemas mentales. De hecho, existen investigaciones que relacionan la percepción de la innovación y el desempeño de docentes y estudiantes al utilizar estrategias didácticas que integren la tecnología. En efecto, la integración de tecnologías en la educación, tales como dispositivos móviles y páginas Web, demandan una transformación de los esquemas mentales del profesorado. Más aún, a raíz de la pandemia del COVID-19, esta percepción del claustro y de la población estudiantil ha cambiado radicalmente, obligando también al sector de la educación de personas ejecutivas a tomar medidas de innovación inmediatas.

A lo largo de los años, clasificaciones como la taxonomía de Bloom -incluyendo la taxonomía revisada y la taxonomía digital- han demostrado su efectividad en la docencia de estudiantes de primaria, secundaria y educación superior. Incluso, existen estudios orientados a explicar la efectividad de la taxonomía de Bloom en equipos de trabajo. El funcionamiento de este tipo de taxonomías consiste en que el razonamiento inductivo (propuesto por la Gestalt) permite alcanzar niveles de abstracción superiores, logrando así un proceso de 
enseñanza-aprendizaje fluido y eficaz. Los modelos de integración en tecnología (SAMR y TPACK) siguen esta misma dinámica inductiva, lo cual permite afirmar que pueden tener un impacto similar al de otras taxonomías más reconocidas.

Asimismo, está demostrado que los modelos SAMR y TPACK habilitan la reflexión y la integración de la tecnología en diversos campos de la enseñanza. De hecho, se ha estudiado que la integración de la tecnología en ciertas temáticas de aprendizaje habilita la integración en otras materias. De este modo, la conceptualización de nuevos modelos de enseñanza en ciertas disciplinas del saber puede habilitar la integración de tecnologías en otros campos académicos, tales como el de personas ejecutivas.

Desafortunadamente, existe poca investigación sobre la integración de la tecnología en personas ejecutivas. Debido a que se ha demostrado el enriquecimiento del aprendizaje de docentes y del estudiantado, gracias al uso de estos modelos, el presente ensayo científico propone que su uso se extienda a la formación de personas adultas con experiencia profesional. En consecuencia, la educación de personas ejecutivas puede facilitarse hasta lograr una adecuada integración de las nuevas tecnologías. Ejercitarse en una mentalidad de transformación y no ya de mejora, podría habilitar que el profesorado integre la tecnología de manera más efectiva, espontánea e incluso, intuitiva.

Lo anterior se puede lograr en tres pasos. En primer lugar, visualizar la tecnología como un recurso -no un fin- para facilitar la entrega de conocimiento, cuyo nivel más elevado se consigue con la abstracción. En segundo lugar, dotar al profesorado de personas ejecutivas de un sistema que le facilite integrar la tecnología en sus procesos de enseñanza, tales como el TPACK y el SAMR. En tercer lugar, explicar que las clasificaciones de los objetivos de aprendizaje siguen una lógica inductiva, la cual es coherente con el estilo de aprendizaje de personas adultas. Considerando esas tres ideas podría lograrse que los modelos de integración de tecnología apoyen eficazmente al profesorado de ejecutivos en su práctica docente.

Si bien es cierto, modelos como el SAMR y el TPACK facilitan la integración de la tecnología en el aula, eso no excluye que otros modelos ya existentes -o incluso nuevossean de provecho al personal docente. No obstante, estos dos son aparentemente los más elaborados y difundidos. Queda pendiente seguir analizándolos, hacer planes piloto, ensayar, incorporarlos dentro de la educación de personas ejecutivas y promoverlos a nivel institucional. Es previsible que se irán difundiendo y poco a poco podrían tomar mayor protagonismo en este sector de la educación. Desgraciadamente, el desarrollo de personas formadoras no es 
la prioridad en muchas instituciones, ni académicas, ni empresariales, y el deseo de innovar y explorar metodologías no es proporcional al esfuerzo que hacen por reinventar la enseñanza y optimizar los recursos que poseen.

El trabajo remoto, el tele trabajo y el aprendizaje en línea son condiciones de la docencia que seguirán avanzando a paso firme. A su vez, los procesos de reactivación y recuperación post COVID-19 no parecieran contemplar que los procesos de enseñanza y aprendizaje sean como antes. Por tanto, próximas investigaciones deberían ir orientadas no solo a entender el comportamiento humano mientras se estudia o enseña virtualmente, sino a comprender estos modelos y construir mejores maneras de integrar la tecnología dentro de la práctica educativa.

\section{Referencias}

Aiyegbayo, Olaojo. (2015). How and why academics do and do not use iPads for academic teaching? British Journal of Educational Technology, 46(6), 1324-1332. doi: https://doi.org/10.1111/bjet.12202

Aldunate, Roberto., y Nussbaum, Miguel. (2013). Teacher adoption of technology. Computers in Human Behavior, 29(3), 519-524. doi: https://doi.org/10.1016/i.chb.2012.10.017

Anastopoulou, Stamatina., Sharples, Mike., y Baber, Chris. (2011). An evaluation of multimodal interactions with technology while learning science concepts. British Journal of Educational Technology, 42(2), 266-290. doi: https://doi.org/10.1111/j.1467-8535.2009.01017.x

Anderson, Lorin., y Krathwohl, David. (2001). A Taxonomy for Learning, Teaching, and Assessing: a Revision of Bloom's Taxonomy of Educational Objectives. New York, United States: Longman.

Arneson, Jessie., y Offerdahl, Erika. (2018). Visual Literacy in Bloom: Using Bloom's Taxonomy to Support Visual Learning Skills. CBE Life Sciences Education, 17(1). doi: https://doi.org/10.1187/cbe.17-08-0178

Baran, Evrim., Canbazoglu Bilici., Sedef., Albayrak Sari, Aylin., y Tondeur, Jo. (2017). Investigating the impact of teacher education strategies on preservice teachers' TPACK. British Journal of Educational Technology, 50(1). doi: https://doi.org/10.1111/bjet.12565

Bloom, Benjamín., Engelhart, Max., Furst, Edward., Hill, Walker., y Krathwohl, David. (1956). Taxonomy of educational objectives: the classification of educational goals. Michigan, United States: David McKay Company.

Brown, Malcom., McCormack, Mark., Reeves, Jamie., Brooks, Christopher., Grajek, Susan., Bali, Maha, ... Weber, Nicole. (2020). 2020 EDUCAUSE Horizon Report TM Teaching and Learning Edition. Louisville: EDUCAUSE

Caird, Sally., y Lane, Andy. (2015). Conceptualising the role of information and communication technologies in the design of higher education teaching models used in the UK. British Journal of Educational Technology, 46(1), 58-70. doi: https://doi.org/10.1111/bjet.12123 
Campos, Roy. (2020). Integración de la tecnología audiovisual en la educación de personas ejecutivas: el profesorado reflexivo. Revista Actualidades Investigativas en Educación, 20(2), 1-31. doi: https://doi.org/10.15517/aie.v20i2.41645

Cejas León, Roberto., Navío Gámez, Antonio., y Barroso Osuna, Julio. (2016). Las competencias del profesorado universitario desde el modelo TPACK (Conocimiento Tecnológico y Pedagógico del Contenido). Pixel-Bit. Revista de Medios y Educación, (49), 105-119. Recuperado de https://www.redalyc.org/pdf/368/36846509008.pdf

Chang, Yahui., Jang, Syh-Jong., y Chen, Yang-Hsueh. (2015). Assessing university students' perceptions of their Physics instructors' TPACK development in two contexts. British Journal of Educational Technology, 46(6), 1236-1249. doi: https://doi.org/10.1111/bjet.12192

Churches, Andrew. (2009). Bloom's Digital Taxonomy. Recuperado de http://burtonslifelearning.pbworks.com/f/BloomDigitalTaxonomy2001.pdf

Dale, Edgar. (1946). Audiovisual methods in teaching. New York, United States of America: Dryden Press.

Dwyer, Christopher., Hogan, Michael., y Stewart, lan. (2014). An integrated critical thinking framework for the 21st century. Thinking Skills and Creativity, 12, 43-52. doi: https://doi.org/10.1016/j.tsc.2013.12.004

Edmondson, Amy., y Mcmanus, Stacy. (2007). Methodological Fit In Management Field Research. Academy of Management Review, 32(4), 1155-1179. Recuperado de https://www.cebma.org/wp-content/uploads/Edmondson-and-McManus-2007-.pdf

Ertmer, Peggy., Ottenbreit-Leftwich, Anne., Sadik, Olgun., Sendurur, Emine., y Sendurur, Polat. (2012). Teacher beliefs and technology integration practices: A critical relationship. Computers and Education, 59(2), 423-435. doi: https://doi.org/10.1016/j.compedu.2012.02.001

Fadel, Charles., y Lemke, Cheryl. (2012). Multimodal Learning through Media. In: N.M. Seel (Ed.) Encyclopedia of the Sciences of Learning. Boston, United States of America: Springer. doi: https://doi.org/10.1007/978-1-4419-1428-6 922

Gebre, Engida., Saroyan, Alenoush., y Bracewell, Robert. (2014). Students' engagement in technology rich classrooms and its relationship to professors' conceptions of effective teaching. British Journal of Educational Technology, 45(1), 83-96. doi: https://doi.org/10.1111/bjet.12001

Gilboy, Mary., Heinerichs, Scott., y Pazzaglia, Gina. (2015). Enhancing student engagement using the flipped classroom. Journal of Nutrition Education and Behavior, 47(1), 109-114. doi: https://doi.org/10.1016/j.jneb.2014.08.008

Glover, lan., Hepplestone, Stuart., Parkin, Helen., Rodger, Helen., y Irwin, Brian. (2016). Pedagogy first: Realising technology enhanced learning by focusing on teaching practice. British Journal of Educational Technology, 47(5), 993-1002. doi: https://doi.org/10.1111/bjet.12425 
Graham, Charles. (2011). Theoretical considerations for understanding technological pedagogical content knowledge (TPACK). Computers \& Education, 57(3), 1953-1960. doi: https://doi.org/10.1016/j.compedu.2011.04.010

Grajek, Susan. (2020). Top 10 IT Issues 2020: The Drive to Digital Transformation Begins. Recuperado de https://er.educause.edu/articles/2020/1/top-10-it-issues-2020-the-drive-todigital-transformation-begins

Hamilton, Ericka., Rosenberg, Joshua., y Akcaoglu, Mete. (2016). The Substitution Augmentation Modification Redefinition (SAMR) Model: a Critical Review and Suggestions for its Use. TechTrends, 60, 443-441. doi: https://doi.org/10.1007/s11528-016-0091-y

Harris, Judith., Mishra, Punya., y Koehler, Matthew. (2009). Teachers' Technological Pedagogical Content Knowledge and Learning Activity Types: Curriculum-Based Technology Integration Reframed. Journal of Research on Technology in Education, 41(4), 393-416. Recuperado de https://files.eric.ed.gov/fulltext/EJ844273.pdf

Hashim, Kamarul., Tan, Felix., y Rashid, Ammar. (2015). Adult learners' intention to adopt mobile learning: A motivational perspective. British Journal of Educational Technology,46(2). doi: https://doi.org/10.1111/bjet.12148

Hilton, Jason. (2016). A Case Study of the Application of SAMR and TPACK for Reflection on Technology Integration into Two Social Studies Classrooms. The Social Studies, 107(2), 68-73. doi: https://doi.org/10.1080/00377996.2015.1124376

Howard, Sarah., Chan, Amy., y Caputi, Peter. (2015). More than beliefs: Subject areas and teachers' integration of laptops in secondary teaching. British Journal of Educational Technology, 46(2), 360-369. doi: https://doi.org/10.1111/bjet.12139

John, Surej. (2015). The integration of information technology in higher education: A study of faculty's attitude towards IT adoption in the teaching process. Contaduría y Administración, 60(S1), 230-252. doi: http://dx.doi.org/10.1016/j.cya.2015.08.004

Koehler, Matthew., y Mishra, Punya. (2009). What Is Technological Pedagogical Content Knowledge ?. Contemporary Issues in Technology and Teacher Education, 9(1), 60-70. Recuperado de https://bit.ly/35tmDpF

Koffka, Kurt. (1935). Principles of Gestalt Psychology. New York, United States of America: Harcourt, Brace \& Company, 1963.

Kolb, Alice., y Kolb, David. (2011a). Experiential Learning Theory: A Dynamic, Holistic Approach to Management Learning, Education and Development. In The SAGE Handbook of Management Learning, Education and Development (pp. 42-68). London: Sage Publications.

Kolb, Alice., y Kolb, David. (2011b). The Kolb Learning Style Inventory 4.0: Guide to Theory, Psychometrics, Research \& Applications. Boston, United States Of America: Hay Resources Direct. 
Kolb, David. (2015). Experiential Learning: Experience as the Source of Learning and Development ( $2^{\text {nd }}$ ed.). New Jersey, United States of America: Pearson Education, Inc. Recuperado de https://bit.ly/2IxN4RW

Kopcha, Theodore., Rieber, Lloyd., y Walker, Brandy. (2016). Understanding university faculty perceptions about innovation in teaching and technology. British Journal of Educational Technology, 47(5), 945-957. doi: https://doi.org/10.1111/bjet.12361

Kotrlik, Joe., y Redmann, Donna. (2005). Extent of Technology Integration in Instruction by Adult Basic Education Teachers. Adult Education Quarterly, 55(3), 200-219. doi: https://doi.org/10.1177/0741713605274630

Krathwohl, David. (2002). A Revision of Bloom's Taxonomy: An Overview. Theory Into Practice, 41(4), 212-218. Recuperado https://www.depauw.edu/files/resources/krathwohl.pdf

Lee, Chia-Jung., y Kim, ChanMin. (2014). The Second Prototype of the Development of a Technological Pedagogical Content Knowledge Based Instructional Design Model: An Implementation Study in a Technology Integration Course. Contemporary Issues in Technology and Teacher Education, 14(3), 297-326. Recuperado de https://www.learntechlib.org/primary/p/114339/

Lindsay, Lucie. (2016). Transformation of teacher practice using mobile technology with one-toone classes: M-learning pedagogical approaches. British Journal of Educational Technology, 47(5), 883-892. doi: https://doi.org/10.1111/bjet.12265

López García, Juan Carlos. (2015). SAMR, modelo para integrar las TIC en procesos educativos. Recuperado de http://eduteka.icesi.edu.co/articulos/samr

Martin, Florence., y Ertzberger, Jeffrey. (2016). Effects of reflection type in the here and now mobile learning environment. British Journal of Educational Technology, 47(5), 932-944. doi: https://doi.org/10.1111/bjet.12327

McClure, Paul. (2018). "You're Fired," Says the Robot: The Rise of Automation in the Workplace, Technophobes, and Fears of Unemployment. Social Science Computer Review, 36(2), 139-156. doi: https://doi.org/10.1177/0894439317698637

Miller, Christine. (2019). Leading Digital Transformation in Higher Education: a toolkit for technology leaders. Pennsylvania, United States of America: IGI Global.

Mishra, Punya., y Koehler, Matthew. (2005). What happens when teachers design educational technology? The development of technological pedagogical content knowledge. Journal of Educational Computing Research,- 32, 131-152. doi: https://doi.org/10.2190\%2F0EW701WB-BKHL-QDYV

Mishra, Punya., y Koehler, Matthew. (2006). Technological pedagogical content knowledge: A framework for teacher knowledge. Teachers College Record, 108(6), 1017-1054. Recuperado de https://bit.ly/36NLynp 
Polanyi, Michael. (1966). The tacit dimension. New York, United States of America: Doubleday \& Company.

Puentedura, Ruben. (2006). Transformation, Technology, and Education [Diapositiva de Pover Point]. Recuperado de http://hippasus.com/resources/tte/

Puentedura, Ruben. (8 de diciembre, 2010). SAMR, TPCK, and Educational Technology: Three Presentations [Diapositiva de Pover Point]. Recuperado de http://www.hippasus.com/rrpweblog/archives/000049.html

Puentedura, Ruben. (11 de diciembre, 2014). SAMR and TPCK: A Hands-On Approach to Classroom Practice [Diapositiva de Pover Point]. Recuperado de http://www.hippasus.com/rrpweblog/archives/000140.html

Scott, Karen. (2013). Does a university teacher need to change e-learning beliefs and practices when using a social networking site? A longitudinal case study. British Journal of Educational Technology, 44(4), 571-580. doi: https://doi.org/10.1111/bjet.12072

Shulman, Lee. (1986). Those who understand: knowledge growth in teaching. Educational Researcher, 15(2), 4-14. doi: https://doi.org/10.3102\%2F0013189X015002004

Sousa, David. (2015). How the brain learns ( $5^{\text {th }}$ ed.). United States of America: Corwin Books.

Spector, J. Michael., Elen, Jan., Merrill, M. David., y Bishop, M. J. (Eds.). (2014). Handbook of research on educational communications and technology $\left(4^{\text {th }}\right.$ ed.). New York, United States of America: Springer.

Tarling, Isabel., y Ng'ambi, Dick. (2016). Teachers pedagogical change framework: a diagnostic tool for changing teachers' uses of emerging technologies. British Journal of Educational Technology, 47(3), 554-572. doi: https://doi.org/10.1111/bjet.12454

Verpoorten, Dominique., Westera, Wim., y Specht, Marcus. (2012). Using reflection triggers while learning in an online course. British Journal of Educational Technology, 43(6), 1030-1040. doi: https://doi.org/10.1111/j.1467-8535.2011.01257.x

Wagemans, Johan., Elder, James., Kubovy, Michael., Palmer, Stephen., Peterson, Mary., Singh, Manish., y von der Heydt, Rüdiger. (2012a). A century of Gestalt psychology in visual perception: I. Perceptual grouping and figure-ground organization. Psychological Bulletin, 138(6),1172-1217. doi: https://doi.org/10.1037/a0029333

Wagemans, Johan., Feldman, Jacob., Gepshtein, Sergei., Kimchi, Ruth., Pomerantz, James., Van der Helm, Peter., y Van Leeuwen, Cees. (2012b). A century of Gestalt psychology in visual perception: II. Conceptual and theoretical foundations. Psychological Bulletin,136(6),1218-1252. doi: https://doi.org/10.1037/a0029334

Wang, Jun., Wei, Wei., Ding, Liting., y Li, Junpeng. (2017). Method for analyzing the knowledge collaboration effect of R\&D project teams based on Bloom's taxonomy. Computers \& Industrial Engineering, 103, 158-167. doi: https://doi.org/10.1016/i.cie.2016.11.010 
Revista indizada en

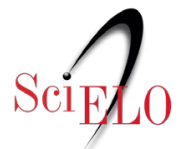

redalyc.sy latindex

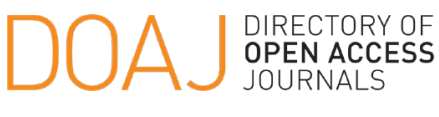

Distribuida en las bases de datos:

- Dialnet

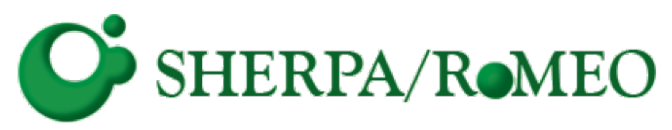

REDIB

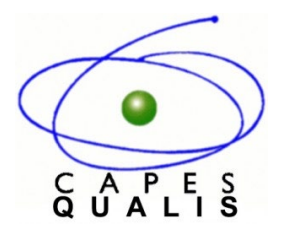

MIAR 